\title{
Infrared Observational Studies of Gas Molecules in Disks
}

\author{
C. Salyk ${ }^{1}$ \\ ${ }^{1}$ The University of Texas, Department of Astronomy, 1 University Station, C1400, Austin, \\ TX 78712 , USA
}

\begin{abstract}
There remain many fundamental unanswered questions about protoplanetary disks, including how (and if?) they form planets, how mass is transferred through the disk and onto the star, and how they ultimately disperse. Also, a major goal of protoplanetary disk studies is to understand the relationship between disk properties and the physical and chemical properties of planetary systems. IR molecular spectroscopy is a particularly powerful tool for probing the conditions and physical process in protoplanetary disks, which are too small and close to their parent stars to be imaged with ease. I will discuss the suite of infrared molecular transitions observed to date, which highlight the following three techniques of IR spectroscopy. Firstly, line shapes and strengths can be used as tracers of disk physics, including volatile condensation/evaporation, photo-processes, grain growth and turbulence. Secondly, observations of multiple molecular abundances provide constraints for disk chemical models, which may ultimately help explain the great diversity of planetary bodies. Finally, resolved line shapes and spectro-astrometry provide a means to study disk structure on extremely small size scales. Because IR observations are typically sensitive to radii of a few AU or smaller, the processes and structures being probed are relevant to the birth and growth of terrestrial and giant planets. Recent results that I will highlight include the discovery of a multitude of molecules in disks around sun-like stars (including $\mathrm{H}_{2} \mathrm{O}, \mathrm{OH}, \mathrm{HCN}, \mathrm{C}_{2} \mathrm{H}_{2}$ and $\mathrm{CO}_{2}$ ), with detection rates that depend on stellar mass, constraints on gas mass and location in transitional disks, detection and characterization of 'snow lines', measurements of inner disk rims, and detections of inner disk asymmetries. I will also discuss how IR spectroscopy will remain relevant even with the emergence of facilities such as ALMA, as it allows us to connect the conditions in terrestrialplanet-forming regions with those in the cold outer reaches of disks, and to better construct a comprehensive understanding of the nature of protoplanetary disks.
\end{abstract}

Keywords. stars: circumstellar material, planets and satellites: formation, infrared: stars

\section{Introduction}

Protoplanetary disks are believed to be the birthplace of planets, and so offer us an opportunity to observe planet formation in progress, and to put our solar system in a larger astronomical context. In particular, studies of protoplanetary disks allow us to ask whether the solar system is a likely or unlikely outcome of the planet formation process. Protoplanetary disks form as the result of angular momentum conservation during the formation of young stars, and so planet formation is intimately linked with star formation. There are numerous star-forming regions within a distance of $\sim 500 \mathrm{pc}$ from the sun and therefore ample opportunity to study such regions. However, investigations are hampered by the small angular separations and high contrast between protostar and disk. This is especially true for terrestrial and gas giant planet-forming regions in disks $(\lesssim 5 \mathrm{AU})$, which are $\lesssim 0.03^{\prime \prime}$ from their parent star at typical cloud distances $(\sim 150 \mathrm{pc})$.

Infrared molecular spectroscopy offers many advantages for overcoming the difficulties involved in observing planet forming regions in disks. Most importantly, in most of the 
infrared regime, the disk emission dominates over the stellar emission, and there is no need to block out the light of the central star. Also, since the disk has vertical and radial temperature gradients, particular molecular transitions nearly always originate in restricted regions of the disk. Thus by observing different transitions, one can obtain spatial information - even though the region is formally spatially unresolved. Finally, since the molecules are subject to Keplerian rotation, Doppler-induced line broadening can be used as a proxy for disk location, and provide further spatial information.

The use of infrared molecular lines to study protoplanetary disks can be roughly separated into the following categories. Firstly, line shapes and strengths can be used as tracers of disk physics, including volatile condensation/evaporation, photo-processes, grain growth and turbulence. Secondly, observations of multiple molecular abundances provide constraints for disk chemical models, which may ultimately help explain the great diversity of planetary bodies. Finally, resolved line shapes and spectro-astrometry provide a means to study disk structure on extremely small size scales. Until $\sim 2008$, such studies were primarily limited to observations of rovibrational CO, but the field has since had a major breakthrough with the discovery that water and organics are commonly observed in the mid-infrared. In this review, I will discuss several molecular tracers in turn, highlighting how each has and can be used to probe particular aspects of protoplanetary disks and planet formation around low-mass (T Tauri; cTT) and mid-mass (Herbig $\mathrm{Ae} / \mathrm{Be}$; HAeBe) stars. I will particularly focus on recent observations and studies, and possible directions for future work.

\section{CO rovibrational emission}

\subsection{First detections}

The CO molecule is an excellent probe of inner protoplanetary disk atmospheres, due to its high abundance and high dissociation temperature. CO overtone emission was one of the first molecular transitions observed around young stars (Scoville et al. 1993) and found to be consistent with a disk origin (Scoville et al. 1983; Carr et al. 1989; Calvet et al. 1991). However, it seemed to be restricted to emission from young sources with strong outflows, with a further possible correlation with higher-mass (HAe/Be) stars (Najita et al. 2000). CO fundamental emission at $\sim 4.6 \mu \mathrm{m}$, on the other hand, was found to be a common feature of the spectra of disks around both T Tauri (Najita et al. 2000; Najita et al. 2003) and Herbig Ae/Be (Blake \& Boogert 2004) stars. An analysis of lineshapes confirmed inner disks (radii $<0.1 \mathrm{AU}$ for cTT disks and $<1 \mathrm{AU}$ for HAeBe disks) as the dominant origin of the emission. These emission lines were strong evidence for a warm disk atmosphere, with a temperature inversion, likely caused by stellar irradiation.

\subsection{Transitional disks}

CO fundamental emission lines have been used to shed light on the properties and origin of transitional disks - disks with inner depletions of small dust grains (extending from the stellar surface to distances between a few and a few tens of AU; Andrews et al. 2011). The cause for the dust depletion is still actively being debated, with possible explanations including photoevaporation from the central star (Hollenbach et al. 1994; Alexander et al. 2007; Ercolano et al. 2008), grain growth, the presence of one or more stellar (Ireland \& Kraus 2008) or sub-stellar (e.g., Rice et al. 2003) companions, or some combination of these effects. One key prediction of the photoevaporation hypothesis is that the inner disk should be cleared of gas as well as dust. The detection of CO fundamental emission from two canonical transitional disks, GM Aur and TW Hya (Rettig et al. 2004; Salyk et al. 2007), suggested the presence of gas in the dust-depleted regions, and lineshape 


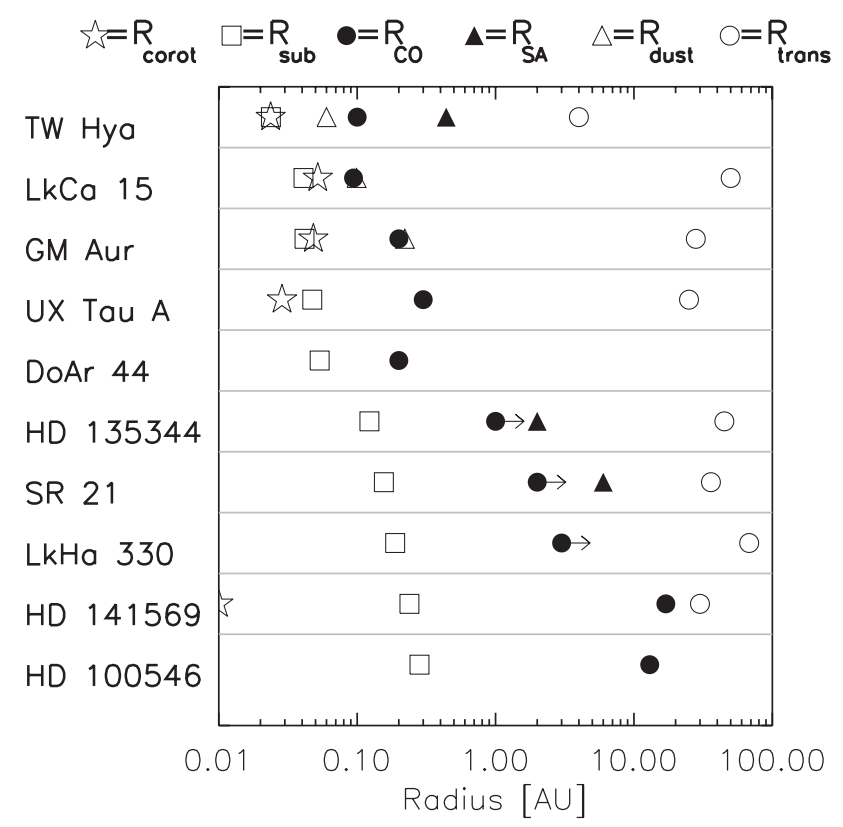

Figure 1. Comparison between $R_{\text {corot }}$ (the corotation radius), $R_{\text {sub }}$ (the dust sublimation radius), $R_{C O}$ (the CO inner radius derived from lineshapes), $R_{\mathrm{SA}}$ (the $\mathrm{CO}$ radius derived from spectro-astrometry), $R_{\text {dust }}$ (the dust inner radius derived from interferometry) and $R_{\text {trans }}$ (the radius at which the disk becomes optically thick). $R_{C O}$ for HD 141569 and HD 100546 are from Brittain et al. (2003) and Brittain et al. (2009), respectively; others are from Salyk et al. (2011b). ( $R_{\mathrm{SA}}$ from Pontoppidan et al. 2008; $R_{\text {trans }}$ from Marsh et al. 2002, Brown et al. 2009, Akeson et al. 2011 and Andrews et al. 2011). Figure from Salyk et al. (2011b).

analysis confirmed that the emission originated at radii $<1$ AU (Salyk et al. 2007). These results have been further supported by spectro-astrometric measurements, which provide a firm upper-limit on the emission radii (Pontoppidan et al. 2008). These results show that some transitional disks were not formed by photoevaporation.

However, the full picture is more complex and interesting. Some transitional disks show CO fundamental emission originating inside the cleared region, but for others the emission originates at or near the wall at which the disk becomes optically thick, and for still others, there is no emission at all (Brittain et al. 2003; Brittain et al. 2009; Salyk et al. 2009). These results suggest that transitional disks are an inhomogeneous class of disks, with some formed by planetary companions, but others formed by stellar companions or photoevaporation (Salyk et al. 2009). A summary of CO emission radii and other observed radii in transitional disks can be seen in Figure 1.

\subsection{Inner disk structure}

Because the molecules in protoplanetary disks undergo (nearly) Keplerian motion, the shape of spectrally resolved emission lines reflect their radial origin. In particular, the emission line wings originate from the innermost part of the molecular disk. An analysis of lineshapes from $\mathrm{T}$ Tauri disks was consistent with emitting radii $<0.1 \mathrm{AU}$, or similar to the stellar corotation radius (Najita et al. 2003) - the approximate radius at which the disk is expected to be truncated due to magnetic interaction with the host star. However, observations of CO fundamental emission from HAeBe disks hinted at a dependence of emitting location on stellar mass, as these emission lines had radial origins in the range 


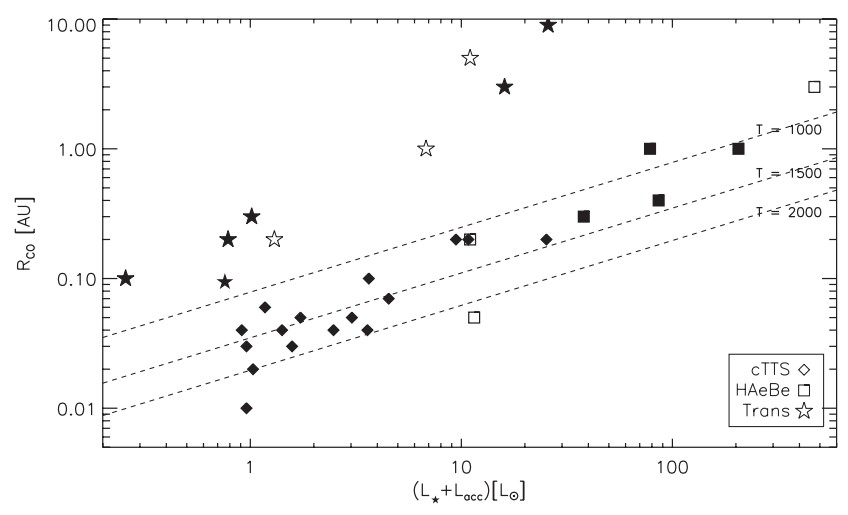

Figure 2. CO inner radius against luminosity. Diamonds are cTTS disks, squares are HAeBe disks and stars are transitional disks. Filled symbols have measured accretion rates, which are incorporated into $L_{\text {acc }}$, while un-filled symbols have $L_{\text {acc }}=0$. Dashed lines show theoretical curves for dust sublimation radius as a function of luminosity. Figure from Salyk et al. (2011b).

of 0.5-1 AU (Blake \& Boogert 2004). A more complete study, spanning several orders of magnitude in luminosity, now shows that the inner radius of the $\mathrm{CO}$ disk scales with stellar luminosity (see Figure 2). The observed trend is similar to a trend observed for the inner extent of dust emission, which is believed to be set by the dust sublimation temperature (see Dullemond \& Monnier 2010 for a review). The CO inner radius might be expected to trace the dust inner radius, since $\mathrm{CO}$ can be easily photodissociated by stellar UV radiation (van Dishoeck \& Black 1988). Interestingly, larger CO radii are derived for transitional disks. An exciting possibility is that this reflects dynamical truncation by a planet, though a more prosaic explanation — that the low dust densities in transitional disks allow the UV to dissociate a larger portion of the disks - is possible.

Inner disk structure is now being probed at an even deeper level with spectroastrometry. With this technique, the centroid of an emission line is measured as a function of velocity, and can be compared with a disk model to measure emitting locations, disk inclinations and disk position angles. Along with firmly establishing an inner disk origin for $\mathrm{CO}$ fundamental emission from both classical and transitional protoplanetary disks, this technique has begun to reveal disk asymmetries at radii of a few AU or less, as well as possible disk warps (Pontoppidan et al. 2011).

\subsection{Disk winds}

Although a majority of $\mathrm{CO}$ fundamental emission from protoplanetary disks can be recreated with a Keplerian disk model (e.g. Najita et al. 2003; Salyk et al. 2011), a significant minority of sources show Lorentzian-like emission profiles that can not be produced with such a model (Bast et al. 2011; Pontoppidan et al. 2011). The lineshapes, measured at extremely high spectral resolution $\left(R \sim 10^{5}\right)$ with VLT-CRIRES, require a significant low-velocity emission component (see Figure 3 ). The source of this component is still under debate, but a leading hypothesis is that the source is a low-velocity disk wind, as described in Figure 3 (Pontoppidan et al. 2011).

\subsection{Istopologues}

Observations of the isotopologues of $\mathrm{CO}$ allow for an investigation of fractionation processes. Taking advantage of the high spectral resolution of CRIRES and a special binary system geometry, Smith et al. (2009) used absorption spectroscopy to measure ${ }^{12} \mathrm{C}:{ }^{13} \mathrm{C}$ and $\mathrm{C}^{17} \mathrm{O}: \mathrm{C}^{18} \mathrm{O}: \mathrm{C}^{16} \mathrm{O}$ ratios for the disk around $\mathrm{VV} \mathrm{CrA}$. They find mass-dependent 


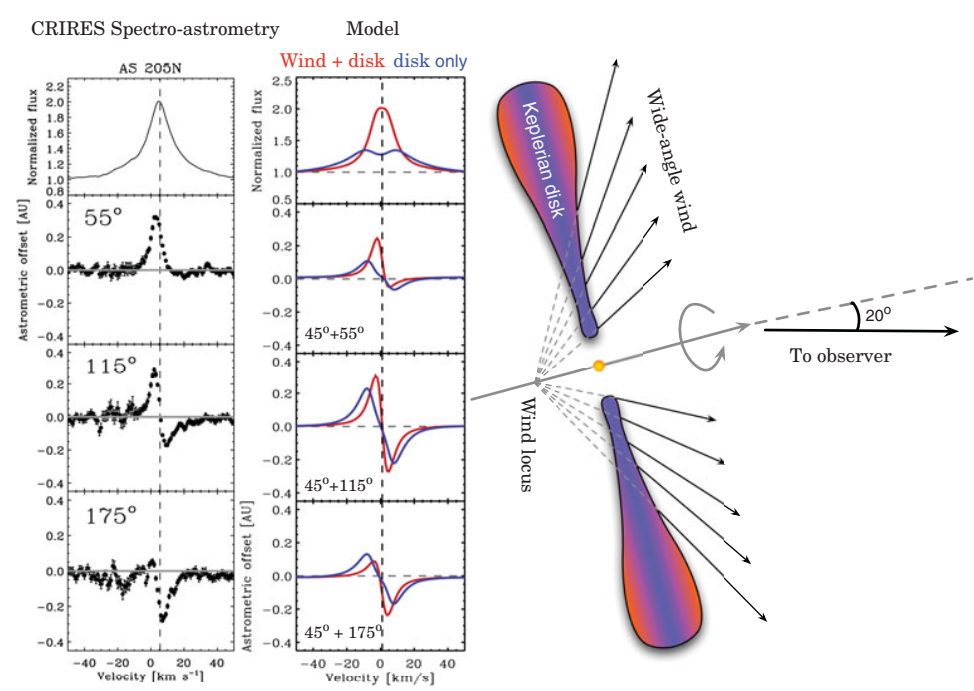

Figure 3. Sketch of a disk wind model that qualitatively reproduces the broad single-peaked CO line spectra and CRIRES spectroastrometry from highly accreting T Tauri stars - in this example, AS 205N, a T Tauri star in Ophiuchus. The left panels show the observed CO rovibrational $(v=1-0$ around $4.7 \mu \mathrm{m})$ line spectrum and spectro-astrometry at 3 different slit position angles. The middle panels show two $2 \mathrm{D}$ radiative transfer models: one for a purely Keplerian disk, and one for a Keplerian disk in which a wide-angle molecular wind is launched from the disk surface. The right panel contains a sketch of the basic wind geometry.Figure from Pontoppidan et al. (2011).

deficits of $\mathrm{C}^{17} \mathrm{O}$ and $\mathrm{C}^{18} \mathrm{O}$, which they suggest is consistent with selective self-shielding - similar to what has been proposed to explain isotopic anomalies in the solar system. They also find a ${ }^{12} \mathrm{C}:{ }^{13} \mathrm{C}$ ratio nearly twice that of the expected Interstellar Medium value, and follow-up observations confirm significant cloud isotopic heterogeneity, which they attribute to ice-gas fractionation (Smith et al. 2010).

\section{3. $\mathbf{H}_{2}$}

$\mathrm{H}_{2}$ is believed to be the most abundant molecular component of circumstellar disks, but as a non-polar molecule, it remains difficult to detect via vibrational and rotational transitions. Rovibrational $(v=1-0 \mathrm{~S}(1))$ emission has been detected by a number of authors (e.g. Weintraub et al. 2000; Bary et al. 2003; Ramsay Howat et al. 2007; Carmona et al. 2008), but spatially resolved observations suggest that the emission lines may be generated via outflow shocks (Beck et al. 2008), rather than in the quiescent disk. In a survey for pure rotational lines from disks around a variety of protostars (spanning classes I-III, with class II including both $\mathrm{HAeBe}$, cTT and transitional disks), $\mathrm{H}_{2}$ was detected only from class I sources, a single HAeBe source and a single class III source (Bitner et al. 2008). The lines are centered at the source velocity, and spectrally and spatially unresolved, suggesting a disk origin at 10-50 AU; however, the results of Beck et al. (2008) show that an outflow origin can not be ruled out. With few infrared detections of $\mathrm{H}_{2}$, and much ambiguity about their origin, infrared $\mathrm{H}_{2}$ lines have not turned out to be a very advantageous probe of protoplanetary disks. 


\section{4. $\mathrm{H}_{2} \mathrm{O}$ and $\mathrm{OH}$}

\subsection{Detections and Origin}

Until recently, detections of water vapor in protoplanetary disks remained elusive. A single unambiguous detection of $\mathrm{H}_{2} \mathrm{O}$ overtone emission was obtained from what turned out to be an atypical disk (Carr et al. 2004), probably representing a younger, more active stage of evolution than a classical $\mathrm{T}$ Tauri disk. In 2008, detections of water, $\mathrm{OH}$ and organics (the last to be discussed in $§ 5$ ) were detected from several more typical cTT disks with the Spitzer Space Telescope InfraRed Spectrograph (Spitzer-IRS; Carr \& Najita 2008; Salyk et al. 2008). Ground-based high-resolution data confirmed an origin in the planet forming (few AU) region (Salyk et al. 2008). This exciting discovery opened up an entirely new avenue of discovery into the study of disks. In particular, as a volatile species actively involved in disk transport and chemistry, water is an ideal molecule for investigating the chemical state and evolution of disks, and condensation processes critical for the growth of giant planet cores.

As a result of a large follow-up survey of disks with Spitzer-IRS, water vapor emission is now known to be a common feature of mid-infrared spectra of cTTs disks (Pontoppidan et al. 2011; see also Figure 4). The richness of this dataset has only begun to be explored.

\subsection{Dependence on system parameters}

Analysis of the large database of Spitzer-IRS spectra immediately showed a striking trend. While detection rates for water and other molecules were greater than $\sim 40 \%$ for cTT disks, these same emission lines were virtually absent from the spectra of HAeBe and transitional disks (Figure 4). Is this an observational bias, due to differing excitation conditions, or due to different chemistry between the two classes of sources? Radiative transfer models predict similar line/continuum ratios for water lines from both cTT and HeAeBe disks if the disks have similar structures and water abundances, so the effect does not appear to be an observational bias (Pontoppidan et al. 2010). The detectability of $\mathrm{CO}$ lines from HAeBe disks shows that line formation is possible, although $\mathrm{CO}$ line/continuum ratios are lower for HAeBe than cTT disks, pointing to at least some difference in line excitation conditions (Salyk et al. 2011a). Also, disk structure does affect the detectability of water lines amongst the cTT population, with detection rate anti-correlated with the 13-30 $\mu \mathrm{m}$ slope - a measure of disk flaring.

However, there is also intriguing evidence for chemical differences between cTT and HAeBe or transitional disks. Bethell \& Bergin (2009) show that the observed water is likely produced in situ and that it can be photodissociated via UV radiation, but is capable of self-shielding. Whether or not the water survives depends on the UV flux, which can be an order of magnitude or more greater for HAeBe disks than cTT disks. Similarly, transitional disks have low dust densities, which leads to less dust shielding from UV, and at least one transitional disk, TW Hya, shows emission from highly excited $\mathrm{OH}$ molecules, which could be produced via photodissociation of water (Najita et al. 2010). Near-IR observations of water vibrational emission also point to chemical differences. While near-IR spectra of cTT disks show water/OH line/continuum ratios near 1, water is undetected in $\mathrm{HAeBe}$ disks, with water/OH line ratios at least an order of magnitude lower (Mandell et al. 2008; Fedele et al. 2011; Figure 5). Such a difference may have profound implications for the chemistry of planets produced in disks around stars with a variety of stellar masses. 


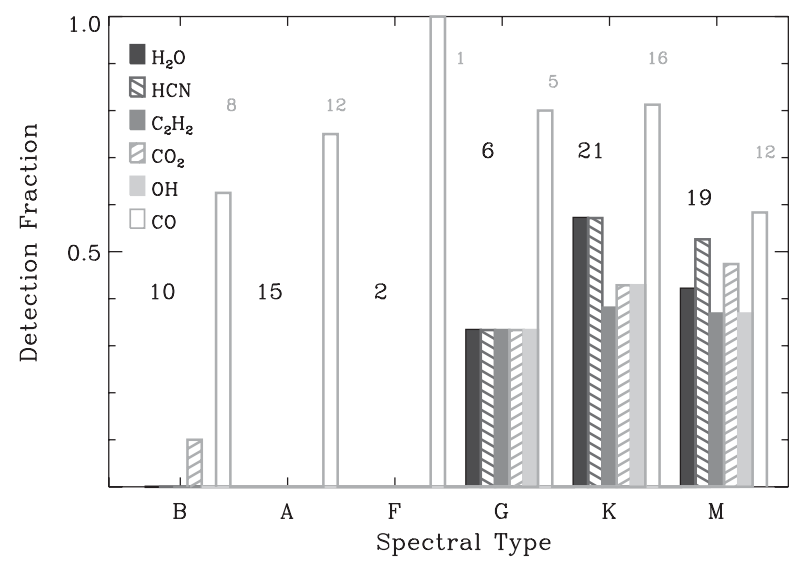

Figure 4. Detection rate as a function of spectral type for several infrared molecular tracers, with the number of each spectral type shown above. The detection rate drops dramatically for spectral types earlier than G/F, except for CO. Figure from Pontoppidan et al. (2010).

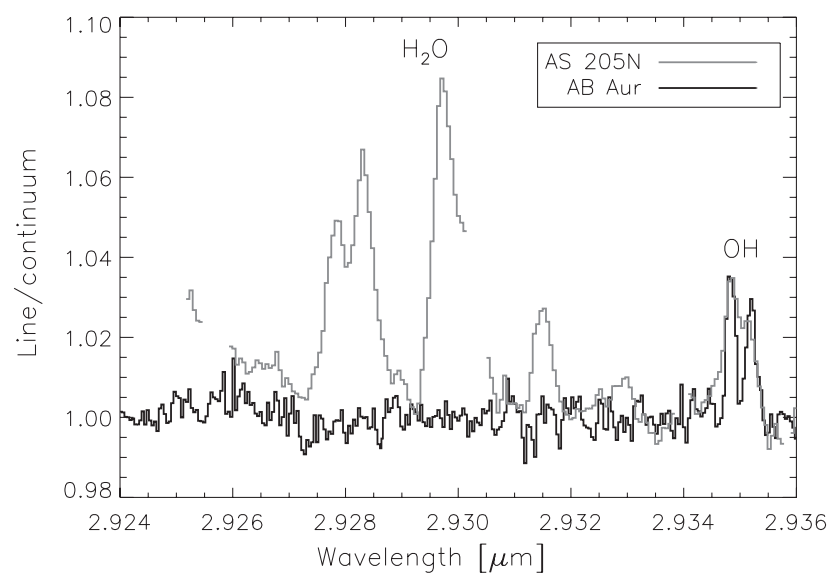

Figure 5. A comparison of water and $\mathrm{OH}$ emission lines observed with Keck-NIRSPEC from AS $205 \mathrm{~N}$ (Salyk et al. 2008) and AB Aur (Mandell et al. 2008), highlighting the radically different water/OH line ratios for $\mathrm{cTT}$ and $\mathrm{HAeBe}$ disks. The $\mathrm{OH}$ line/continuum ratio for $\mathrm{AB}$ Aur was scaled to match that of AS $205 \mathrm{~N}$.

\subsection{Water photochemistry, condensation and transport}

One major goal for the study of water in protoplanetary disks is to understand its chemistry, condensation and transport, and their relation to the growth of planetesimals and the delivery of water to the proto-Earth. Infrared spectra - specifically, the relative strength of low- and high-excitation lines - can be used to determine the radial extent of water vapor, and hence the radius of water ice condensation (the so-called snow-line). This is a crucial parameter for models of planetary system formation, as ice may be a necessary ingredient to form cores large enough to contract a large gaseous envelope (as in the case of Jupiter or Saturn). A qualitative examination of the Spitzer spectra shows a relative dearth of flux from low-excitation lines, and points to a snow-line at only $\sim 1$ AU — well inside the canonical solar system snow-line of 5 AU (Meijerink et al. 2009). A more quantitative exploration of this measurement is in order, and will be greatly 
facilitated by the combination of Spitzer-IRS with far-IR spectra from the Herschel Space Observatory, since the relative line fluxes in the mid- and far-IR are very sensitive to the location of the snow-line.

\section{Other Molecules}

The detection of more complex molecules is necessary to test our understanding of disk chemistry and its relation to the great diversity of planets in our solar system. Sophisticated chemical models, incorporating dozens of molecular and atomic species (e.g. Markwick et al. 2002; Agundez et al. 2008; Willacy et al. 2009; Woitke et al. 2009) have been developed, but the observations necessary to test their conclusions are still in early stages. Infrared features of $\mathrm{C}_{2} \mathrm{H}_{2}, \mathrm{HCN}$ and $\mathrm{CO}_{2}$ were first discovered in absorption by Lahuis et al. (2006) and provided evidence for high temperature chemistry. However, their detection required the uncommon geometry of a nearly edge-on disk. $\mathrm{C}_{2} \mathrm{H}_{2}, \mathrm{HCN}$ and $\mathrm{CO}_{2}$ were finally found in emission by Carr \& Najita, and more extensive surveys have confirmed that such emission is a common feature of the spectra of cTT disks (Pontoppidan et al. 2010; Carr et al. 2011).

Although a wide variety of molecular line strength ratios are observed in cTT disks (Salyk et al. 2011a; Carr \& Najita 2011), the causes of the variation have remained elusive, as line ratios do not clearly correlate with system variables. One possible exception is the observation that the highest $\mathrm{HCN} / \mathrm{H}_{2} \mathrm{O}$ flux ratios are observed in the most massive disks, perhaps because planetesimals have formed, sequestered $\mathrm{O}$, and created a region of Carbon-rich chemistry (Carr \& Najita 2011). Additionally, Teske et al. (2011) note a correlation between HCN fluxes and both stellar accretion rates and X-ray luminosities. These results are all preliminary, and some care must be taken to distinguish cause and effect from covariance. Nevertheless, such studies hold much promise for the near future.

Chemical differences have also been observed between solar-like and cool stars, based on a survey with the Spitzer-IRS low-resolution modules (Pascucci et al. 2009). Cool stars display a significant underabundance of $\mathrm{HCN}$ relative to $\mathrm{C}_{2} \mathrm{H}_{2}$, which the authors propose originates from a difference in UV irradiation. Such a result is another demonstration that stellar mass can have a significant effect on disk chemistry, and possibly influence the types of planets that can be produced around any given star.

\section{Future Directions}

\subsection{In-depth analysis of existing datasets}

A number of rich datasets of molecular spectra have already been obtained, including datasets from Keck-NIRSPEC, VLT-CRIRES, Gemini-Michelle and Spitzer-IRS. These data have been qualitatively examined, and often studied with the use of LTE models. However, given the preponderance of recently acquired high-quality data, there is certainly great science to be derived from existing datasets. A few of the outstanding questions that may still be answered with existing datasets include: Is a low-velocity disk wind the correct explanation for the anomalous, strongly-peaked CO line profiles (Bast et al. 2011; Pontoppidan et al. 2011), and if so, what are its properties? Are molecular lines time variable, and if so, what does that tell us about the nature of the planetforming environment, and the validity of quiescent disk models? What is the cause of variations in line ratios from organic molecules observed in Spitzer-IRS spectra? How do chemical models match up quantitatively with existing data, and what does this tell us about the relevance of various chemical processes? 


\subsection{Improvements in spectral resolution and sensitivity}

Many improvements in spectral resolution, sensitivity and wavelength coverage will be made possible in the coming years via upgrades to existing facilities as well as new facilities and instruments. Combining higher spectral resolution data with the existing Spitzer archive will reduce many modeling degeneracies and make these data more powerful. Improved sensitivity (such as with the James Webb Space Telescope) will allow for the discovery of more molecular species, improving our understanding of chemical pathways and the planet formation environment. It will also allow studies to probe more distant clusters, and fainter stars, such as brown dwarfs, and to search for remnant gas in more evolved disks. Finally, high-resolution instruments with enhanced wavelength coverage will make it possible to conduct large surveys for undiscovered molecules, and achieve simultaneous observations of many species.

\subsection{The time domain}

Time variations of molecular emission features are essentially unstudied, and can easily be explored by combining new and existing datasets. One question upon which such studies would shed light is the origin and nature of the proposed low-velocity disk wind (Pontoppidan et al. 2011). Also, the presence of disk structure variability could have profound impacts for forming protoplanets. Finally, variability could be the result of warps and asymmetric structures caused by the interaction of the disk with embedded protoplanets.

\subsection{Spatial distribution of chemistry}

The combination of existing data with data from current and future facilities - notably the Herschel Space Observatory and ALMA — will allow us to spatially map the distribution of molecules in disks. Herschel is already allowing us to map the location of ice lines in disks with Spitzer-IRS water emission. ALMA will expand this possibility to other volatile species. Roughly speaking, near-IR spectra provide chemistry at $\sim 1$ AU or smaller, while Spitzer-IRS spectra provide a view of the chemistry at radii of a few AU. ALMA, on the other hand, will spatially resolve size scales of a few AU with its largest baseline, and with a sensitivity greatly exceeding that of all existing sub-millimeter or millimeter wave facilities. Thus, for the first time it will be possible to map the abundance of molecules throughout protoplanetary disks. This will bring us closer than ever before to understanding the origin of the physical and chemical diversity of planetary systems.

\section{References}

Agúndez, M., Cernicharo, J., \& Goicoechea, J. R. 2008, A\&\&A, 483, 831

Akeson, R. L., et al. 2011, ApJ, 728, 96

Alexander, R. D. \& Armitage, P. J. 2007, MNRAS, 375, 500

Andrews, S. M., Wilner, D. J., Espaillat, C., Hughes, A. M., Dullemond, C. P., McClure, M. K., Qi, C., \& Brown, J. M. 2011, ApJ, 732, 42

Bast, J. E., Brown, J. M., Herczeg, G. J., van Dishoeck, E. F., \& Pontoppidan, K. M. 2011, $A \& A, 571,119$

Bethell, T. \& Bergin, E. 2009, Science, 326, 1675

Bitner, M. A., et al. 2008, ApJ, 688, 1326

Blake, G. A. \& Boogert, A. C. A. 2004, ApJ(Letters), 606, L73

Brittain, S. D., Rettig, T. W., Simon, T., Kulesa, C., DiSanti, M. A., \& Dello Russo, N. 2003, ApJ, 588, 535

Brittain, S. D., Najita, J. R., \& Carr, J. S. 2009, ApJ, 702, 85 
Brown, J. M., Blake, G. A., Qi, C., Dullemond, C. P., Wilner, D. J., \& Williams, J. P. 2009, ApJ, 704, 496

Calvet, N., Patino, A., Magris, G. C., \& D’Alessio, P. 1991, ApJ, 380, 617

Carmona, A., et al. 2008, A\&A, 477, 839

Carr, J. S. 1989, ApJ, 345, 522

Carr, J. S., Tokunaga, A. T., \& Najita, J. 2004, ApJ, 603, 213

Carr, J. S. \& Najita, J. R. 2008, Science, 319, 1504

Carr, J. S. \& Najita, J. R. 2011, ApJ, 733, 102

Dullemond, C. P. \& Monnier, J. D. 2010, ARAA, 48, 205

Ercolano, B., Drake, J. J., Raymond, J. C., \& Clarke, C. C. 2008, ApJ, 688, 398

Fedele, D., Pascucci, I., Brittain, S., Kamp, I., Woitke, P., Williams, J. P., Dent, W. R. F., \& Thi, W.-F. 2011, ApJ, 732, 106

Hollenbach, D., Johnstone, D., Lizano, S., \& Shu, F. 1994, ApJ, 428, 654

Ireland, M. J. \& Kraus, A. L. 2008, ApJ(Letters), 678, L59

Lahuis, F., et al. 2006, ApJ(Letters), 636, L145

Mandell, A. M., Mumma, M. J., Blake, G. A., Bonev, B. P., Villanueva, G. L., \& Salyk, C. 2008, $A p J$ (Letters), 681, L25

Markwick, A. J., Ilgner, M., Millar, T. J., \& Henning, T. 2002, A\&A, 385, 632

Marsh, K. A., Silverstone, M. D., Becklin, E. E., Koerner, D. W., Werner, M. W., Weinberger, A. J., \& Ressler, M. E. 2002, ApJ, 573, 425

Meijerink, R., Pontoppidan, K. M., Blake, G. A., Poelman, D. R., \& Dullemond, C. P. 2009, ApJ, 704, 1471

Najita, J. R., Edwards, S., Basri, G., \& Carr, J. 2000, Protostars and Planets IV, 457

Najita, J., Carr, J. S., \& Mathieu, R. D. 2003, ApJ, 589, 931

Najita, J. R., Carr, J. S., Strom, S. E., Watson, D. M., Pascucci, I., Hollenbach, D., Gorti, U., \& Keller, L. 2010, ApJ, 712, 274

Pascucci, I., Apai, D., Luhman, K., Henning, T., Bouwman, J., Meyer, M. R., Lahuis, F., \& Natta, A. 2009, ApJ, 696, 143

Pontoppidan, K. M., Blake, G. A., van Dishoeck, E. F., Smette, A., Ireland, M. J., \& Brown, J. 2008, ApJ, 684, 1323

Pontoppidan, K. M., Salyk, C., Blake, G. A., Meijerink, R., Carr, J. S., \& Najita, J. 2010, ApJ, 720,887

Pontoppidan, K. M., Salyk, C., Blake, G. A., \& Käufl, H. U. 2010, ApJ(Letters), 722, L173

Pontoppidan, K. M., Blake, G. A., \& Smette, A. 2011, ApJ, 733, 84

Ramsay Howat, S. K. \& Greaves, J. S. 2007, MNRAS, 379, 1658

Rettig, T. W., Haywood, J., Simon, T., Brittain, S. D., \& Gibb, E. 2004, ApJ, 616, L163

Rice, W. K. M., Wood, K., Armitage, P. J., Whitney, B. A., \& Bjorkman, J. E. 2003, MNRAS, 342, 79

Salyk, C., Blake, G. A., Boogert, A. C. A., \& Brown, J. M. 2007, ApJ(Letters), 655, L105

Salyk, C., Pontoppidan, K. M., Blake, G. A., Lahuis, F., van Dishoeck, E. F., \& Evans, N. J., II 2008, ApJ(Letters), 676, L49

Salyk, C., Blake, G. A., Boogert, A. C. A., \& Brown, J. M. 2009, ApJ, 699, 330

Salyk, C., Pontoppidan, K. M., Blake, G. A., Najita, J. R., \& Carr, J. S. 2011, ApJ, 731, 130

Salyk, C., Blake, G. A., Boogert, A. C. A., \& Brown, J. M., 2011, submitted

Scoville, N., Kleinmann, S. G., Hall, D. N. B., \& Ridgway, S. T. 1983, ApJ, 275, 201

Smith, R. L., Pontoppidan, K. M., Young, E. D., Morris, M. R., \& van Dishoeck, E. F. 2009, ApJ, 701, 163

Smith, R. L., Pontoppidan, K. M., Young, E. D., \& Morris, M. R. 2010, Disks, Meteorites, Planetesimals, 6012

Teske, J. K., Najita, J. R., Carr, J. S., Pascucci, I., Apai, D., \& Henning, T. 2011, arXiv:1104.0249 van Dishoeck, E. F. \& Black, J. H. 1988, ApJ, 334, 771

Willacy, K. \& Woods, P. M. 2009, ApJ, 703, 479

Woitke, P., Kamp, I., \& Thi, W.-F. 2009, A\&A, 501, 383 


\section{Discussion}

LYONS: For observations from $\sim 1$ AU, how high above the midplane is being probed?

SALYK: The emitting region is very small, and most likely limited by the dust opacity. This brings to light the important point that most infrared observations probe only the upper atmosphere and do not necessarily represent the bulk chemistry. Instead, observations must be considered in the context of a full disk model.

LAHUIS: Besides being chemically sensitive, the mid-IR features are also (very) sensitive to perturbations in the inner disk. This is shown by EX-Lupi and IRS $46 \mathrm{C}_{2} \mathrm{H}_{2}$ and HCN being very sensitive - more so than $\mathrm{H}_{2} \mathrm{O}$. This makes the interpretation of the mid-IR spectra even more interesting.

SALYK: Sure.

Semenov: What makes the lines non-Keplerian? Is it turbulence or asymmetries in inner disk regions (e.g. clumpiness, spiral arms)? Many young stars show rapid luminosity variations at optical wavelengths $(\sim$ hours - weeks timescales).

SALYK: The leading hypothesis right now is a slow molecular disk wind, although the current model is not physical, but rather a simple parametric model. The observational constraint is that the model must produce a large flux with low velocities, centered at the stellar velocity. We are looking into this further, including pursuing observations of variability. 